\title{
Monitoring swelling and deswelling of thin polymer films by microcantilever sensors
}

\author{
Mercedes del Rey ${ }^{1}$, Rubens A. da Silva ${ }^{1,2}$, David Meneses ${ }^{1}$, Denise F. S. Petri ${ }^{2 *}$, Javier \\ Tamayo $^{1}$, Montserrat Calleja ${ }^{1}$, Priscila M. Kosaka ${ }^{1 *}$
}

1 -Instituto de Microelectrónica de Madrid, CSIC, Isaac Newton 8 (PTM), Tres Cantos, 28760

Madrid, Spain.

*Corresponding author: priscila@imm.cnm.csic.es

2 - Instituto de Química - Universidade de São Paulo, Brazil, Av. Prof. Lineu Prestes 748, 05508900 São Paulo, Brazil

*Corresponding author: $\underline{\text { dfsp@usp.br }}$ 


\section{Abstract}

Microcantilever based sensors are promising devices because they are fast, low cost, portable and allow measuring extremely small quantities of substances in gas or liquid phase that are not detectable by other methods. In this work we investigate the swelling and deswelling behavior of poly(hydroxyl ethyl methacrylate), PHEMA, films with three different average molecular masses deposited onto bare silicon microcantilevers, a hydrophilic substrate, and onto polystyrene (PS) coated microcantilevers, which is a hydrophobic substrate, under humidity cycles by monitoring the deflections resulting from differential surface stress. The results found in the present study demonstrate that the micromechanical response observed are related not only to the polymer molecular weight, but also to the polymer-interface phenomena and environment -polymer interface that can play a crucial role on the signal transduction. Ellipsometric measurements evidenced that the swelling of PHEMA films with intermediate molecular weight at $80 \% \mathrm{RH}$ follows Fickian diffusion mechanism. Nevertheless, due to the weak optical contrast or film homogeneity shorter or longer chains than PHEMA300 could not be investigated by ellipsometry. PHEMA films deposited onto PS surfaces presented dewetting, which limited the ellipsometric measurements. In comparison to ellipsometry, monitoring swelling behavior of PHEMA by nanomechanical responses is more advantageous because it is faster and less restricted.

Keywords: microcantilevers; poly(hydroxyl ethyl methacrylate); polystyrene; molecular weight; surface stress; humidity; ellipsometry 


\section{Introduction}

Microcantilever $(\mathrm{MC})$ based sensors are promising devices because they are fast, labelfree, low cost, portable and allow measuring extremely small quantities of substances in gas or liquid phase that are not detectable by other methods [1, 2]. The main mechanical effects observed in MCs are surface stress, which causes static mechanical bending, and changes in the resonance frequency $[3,4]$. The surface stress mechanical effect takes place, if only one of the both $\mathrm{MC}$ sides is coated with a film, this operation mode can be designed to follow the mechanical response in real time. If the top side of the $M C$ is coated and the film expands, which might occur due to hydration, favorable/specific interactions or thermal transitions, the cantilever bends away from the coated side, resulting in compressive stress. On the contrary, as the film contracts, the cantilever bends towards the coated side, generating tensile stress. The dynamic response mode is characterized by resonance frequency changes caused by added mass of a given analyte on any of the sensor surfaces. Scheme 1 represents both operation modes of nanomechanical sensors.

In the field of biosensors, MCs have been used for the detection of specific bindings to proteins [5], DNA hybridization $[6,7,8]$ or antibody-antigen interactions $[9,10,11]$. MC sensors can also be used for monitoring viscoelastic changes in thin polymer layers deposited on the upside of MCs. Jung and co-workers [12] used PS-coated silicon cantilevers to determine the thermomechanical and physical properties of the PS sample in the vicinity of the glass transition temperature. They found that the nanomechanical thermal analysis technique using polymer-coated silicon cantilevers can serve as a promising method to experimentally probe the volume expansion and contraction of amorphous polymers in the glassy state upon heating. Recently, the glass transition temperature $\left(T_{g}\right)$ was determined for ultrathin films of poly(3-thiophene methyl acetate), P3TMA, deposited onto MC as $5.2{ }^{\circ} \mathrm{C}$ higher than the $T_{g}$ value determined for the bulk polymer. Such effect was attributed to ordered molecular arrangement at polymer-air interface induced by the nanometer scale thickness [13]. The swelling and deswelling behavior of poly(methyl methacrylate), PMMA, brushes attached to $M C$ was investigated in a fluid cell, where cantilever bending was monitored as solvent/non-solvent mixtures were injected [14]. Polymers can also be swollen by solvent vapor. The usual techniques to investigate swelling behavior of thin polymer layers by a given solvent vapor are reflectometry [15], gravimetry [16], quartz crystal microbalance with dissipation monitoring (QCM-D) [17], attenuated total reflection infrared (ATR-IR) 
spectroscopy [18], optical interferometry [19] and AFM force versus distance interaction curves $[20,21]$. The use of bending responses of polymer coated MC under variable moisture levels has been used to understand the swelling behavior of polymeric networks with different crosslinking degrees [22] or to determine the diffusion coefficient of water in the thin polymer film [23].

Poly(hydroxyl ethyl methacrylate), PHEMA, a polymer widely used for contact lenses, is water-insoluble, but it swells in water or water vapor. The PHEMA based contact lenses should be always hydrated in order to avoid uncomfortable corneal dessication. Opdahl and co-workers investigated the surface stiffness and surface elastic modulus values of commercial crosslinked PHEMA hydrogel by means of AFM force versus distance interaction curves in the $\mathrm{RH}$ range of $36 \%$ to $87 \%[20,21]$. They found that at low humidity $(<60 \% \mathrm{RH})$, the air exposed surface is dehydrated in comparison to the bulk, it is stiffer and behaves as a glassy polymer, whereas at high humidity ( $>60 \% \mathrm{RH}$ ) water acts as a plasticizer, the surface softens and the viscous deformation increases $[20,21]$.

In this work we investigate the swelling and deswelling behavior of PHEMA films deposited onto bare MC, which is a hydrophilic substrate composed of a native layer of $\mathrm{SiO}_{2}$, or onto polystyrene (PS) coated MC, which is a hydrophobic substrate, under humidity cycles of $8 \mathrm{~min}, 15 \mathrm{~min}$ or $60 \mathrm{~min}$, by monitoring the deflections resulting from differential surface stress. PHEMA with three different molecular weights $(20 \mathrm{~kg} / \mathrm{mol}, 300 \mathrm{~kg} / \mathrm{mol}$ and 1000 $\mathrm{kg} / \mathrm{mol}$ ) were deposited on the upside of MCs. Thus the micromechanical effects explored in the present study are related not only to the environment-polymer interface but also to the polymer-substrate interface phenomena and to the polymer molecular weight. Ellipsometric measurements were used to monitor thickness and index of refraction of PHEMA films spincoated onto Si wafers and PS coated Si wafers in the RH range of $25 \%$ to $80 \%$ for periods of time ranging from $5 \mathrm{~min}$ to $4 \mathrm{~h}$.

\section{Experimental}

\subsection{Materials}

Poly(hydroxylethyl methacrylate) (PHEMA) samples with nominal viscosity average molar masses (Mv) 20,000 g.mol ${ }^{-1}$ (PHEMA-20), 300,000 g.mol ${ }^{-1}$ (PHEMA-300) and 1,000,000 g.mol ${ }^{-1}$ (PHEMA-1000), polystyrene (PS) with Mv 300,000 g.mol ${ }^{-1}$, toluene and methanol analytical grade were purchased from Sigma-Aldrich (USA). PHEMA and PS solutions were 
prepared at $30 \mathrm{~g} \mathrm{~L}^{-1}$ in methanol and toluene, respectively, in a sonication bath during 5 minutes.

\subsection{Methods}

Silicon microcantilevers (MC) (Mikromasch, Estonia) arrays rinsed with piranha solution were used as mechanical transducers. The cantilevers are $400 \mu \mathrm{m}$ long, $100 \mu \mathrm{m}$ wide and $1 \mu \mathrm{m}$ thick. The variations of resonance frequencies within one array were less than $1 \%$, indicating similar mechanical properties. Polymer solution at $30 \mathrm{~g} / \mathrm{L}$ was deposited on the MC by spin coating (SÜSS Microtec Delta +10 ), $\omega=10,0000 \mathrm{rpm}, 30 \mathrm{~s}$ ). In the case of PHEMA-300, films were deposited not only on bare MCs, but also on MCs previously coated with a thin PS film ( $4 \mathrm{~nm}$ thick); these films were coded as PS/PHEMA-300. The polymer films were homogeneously deposited on the cantilevers (Figure 1). One should note that less concentrated polymer solutions were not adequate because they led to discontinuous polymer films and would not generate a uniform stress. PHEMA or PS was removed from the MC backside by plasma treatment $\left(80 \mathrm{~W}, 7.5 .10^{-9}\right.$ torr, $\mathrm{O}_{2}$ at $20 \mathrm{sccm}$, RIE Oxford Instruments Plasmalab 80 PLUS), because otherwise there is no cantilever deflection. One should note that plasma treatment caused no crosslinking, film thinning, or changes in surface roughness (Supplementary Information-Figure S1).

The resonance frequency and deflection of polymer coated MC were recorded as a function of air relative humidity $(\mathrm{RH})$ in the experimental setup depicted in Figure 2 . The nanomechanical response of the MC was measured by using a home-built apparatus equipped with an optical readout-technique and the automated two-dimensional scanning of a single laser beam by voice-coil actuators [24]. The beam of a $3 \mathrm{~mW}$ red laser diode (Edmund Optics) was focused on the $\mathrm{MC}$ end and the reflected beam was collected by a two-dimensional position sensing linear detector (PSD). The apparatus has an environmental chamber that allows the control of temperature $(\mathrm{T})$ and $\mathrm{RH}$. The $\mathrm{RH}$ control system is implemented with a humidity probe from Hygrosens (sensor) and two mass-flow controllers (MFC) from Aalborg. The measurement chamber has an inlet for dry/wet $\mathrm{N}_{2}$. One of the MFCs provides a flow of dry $\mathrm{N}_{2}$, and the other one provides a flow of wet $\mathrm{N}_{2}$. Wet $\mathrm{N}_{2}$ is obtained by passing the gas through a wash flask filled with distilled water. Both gas lines are mixed in a pre-chamber before reaching the measurement chamber. The temperature control system was implemented with a standard PT 100 resistance temperature detector located close to the microcantilever array in the measurement chamber and a cooling circulator (Huber Kaeltemaschinenbau $\mathrm{GmbH}$ ) as shown in Figure 2. 
For the humidity cycles, the relative humidity was varied from $0 \%$ to $80 \%$, while the temperature was kept at $25 \pm 1 \stackrel{\circ}{\circ}$. The humidity cycles were completed in $8 \mathrm{~min}, 15 \mathrm{~min}$ or 60 min. The resonance frequency of the microcantilevers was measured at $0 \%$ and $80 \% \mathrm{RH}$ after the stabilization of the environment in the measurement chamber at the desired $\mathrm{RH}$ for 10 minutes.

Silicon wafers (University Wafers, USA) were cut into $1.0 \mathrm{~cm} \times 1.0 \mathrm{~cm}$ pieces and rinsed with piranha solution and deionized water and afterwards dried under $\mathrm{N}_{2}$ stream. Half of the $\mathrm{Si}$ wafer area was covered with a tape resistant to toluene and methanol. Polymer solutions were deposited onto Si wafers by spin coating (SÜSS Microtec Delta +10 ), $\omega=10,000 \mathrm{rpm}, 30$ s. The tape was removed and the mean thickness values of polymer films were determined by means of profilometry (Veeco Dektak 150). The measurement was performed at least at three different points of two different samples.

Ellipsometric measurements were performed with a vertical computer-controlled DREEL02 ellipsometer (Ratzeburg, Germany) with an angle of incidence $\varphi$ of $70.0^{\circ}$ and wavelength $\lambda$ of $632.8 \mathrm{~nm} \mathrm{He}-\mathrm{Ne}$ laser. The mean thickness and index of refraction of the PHEMA layer were determined independently using the fundamental ellipsometric equation [25]:

$$
e^{i \Delta} \tan \psi=\left(R_{p} / R_{s}\right)=f\left(n_{k}, d_{k}, \lambda, \phi\right)
$$

where $R_{p}$ and $R_{s}$ are the overall reflection coefficients for parallel and perpendicular waves, respectively. The mean thickness $d_{k}$ and refractive index $n_{k}$ can be calculated from the changes of the ellipsometric angles $\Delta$ and $\Psi$, using iterative calculations and a multilayer model composed by the substrate, the unknown layer, and surrounding medium. The thickness of the $\mathrm{SiO}_{2}$ layer was determined in air, considering the indices of refraction for $\mathrm{Si}$ as $\mathrm{n}_{\mathrm{si}}=(3.88-$ i0.018) [26], $\mathrm{n}_{\mathrm{air}}=1.00$ and $\mathrm{n}_{\mathrm{SiO} 2}=1.462$ [26] and "infinite" thickness for the Si and air layers. The mean thickness obtained for $\mathrm{SiO}_{2}$ was $(2.0 \pm 0.1) \mathrm{nm}$. The mean thickness and index of refraction determined in air for PS films deposited onto Si wafers amounted to (136 \pm 5$) \mathrm{nm}$ and $(1.587 \pm 0.004)$, respectively. The PHEMA spin-coated onto Si wafers of PS coated Si wafers were inserted in a special cell made of poly(methyl methacrylate) and quartz windows with inclination of $70^{\circ}$ [27]. The $\mathrm{RH}$ inside the ellipsometric cell was controlled by mixing dry $\mathrm{N}_{2}$ and humid $\mathrm{N}_{2}$ and measured by a humidity/ temperature meter Lutron HT 3006A. In the first set of experiments a desired $\mathrm{RH}$ value was adjusted inside the cell and the ellipsometric angles were measured after $4 \mathrm{~h}$. In the second set of experiments the $\mathrm{RH}$ value was constant and the ellipsometric angles were measured as a function of time. All measurements were performed in a conditioned room with temperature at $(24.0 \pm 0.5){ }^{\circ} \mathrm{C}$. 


\section{Results and Discussion}

The procedure for polymer coating of MCs must consider the fragile suspended mechanical structures to avoid changes in the mechanical properties of the cantilever. Several methods can be used for coating MCs with homogeneous polymer layers, but the choice depends on the system. For instance, the ink-jet deposition method was used for poly(vinyl alcohol) depositions, while for poly(vinyl butyral-co-vinyl alcohol-co-vinyl acetate), and poly(vinyl chloride-co-vinyl acetate-co-2-hydroxypropyl acrylate) microarray spotting pins were applied [28]. Polymers can be polymerized onto MCs by plasma polymerization [22,23] or MCs can be functionalized to enable grafting of polymer brushes $[14,29]$ or polymer colloidal particles [30]. In this work PHEMA was spin-coated on bare MCs and PS coated MCs and polymer residues were removed from the $M C$ backside by plasma treatment. The reasons to choose this technique to coat the MC surfaces are (i) the necessity to coat only one side of the $M C$ for the surface stress measurements and (ii) the quality and reproducibility of the polymer film acquired after the spin coating. As shown in typical optical micrographs in Figure 1, continuous and homogeneous polymer films were deposited onto MCs using a very fast and simple method.

The $M C$ deflection setup used in the present work is schematically represented in Figure 2. Basically it consists of one side coated MCs, that act as mechanical transducers, and a deflection readout system confined in a chamber with controlled temperature and air relative humidity (RH). Figure 3a shows typical deflections of PHEMA-20, PHEMA-300 and PHEMA1000 coated MCs as a function of RH cycles at $(25 \pm 1)^{\circ} \mathrm{C}$. As reference, the deflection of PS coated MCs with RH cycles were also recorded (Figure 3a). PS was chosen as reference due to its hydrophobicity.

The deflections result from differential surface stress generated between opposite sides of MC. Upon increasing RH, PHEMA chains swell, increasing the viscoelasticity $[19,21]$ and the osmotic pressure $[29,30]$. This effect causes compressive stress on the coated MC side and, consequently, MCs bend away from the coated side. Figure 3 shows clearly that increasing $\mathrm{RH}$ values from $0 \%$ to $80 \%$ the bending of PHEMA coated MCs increased with polymer molecular weight decrease (PHEMA-20 > PHEMA-300 > PHEMA-1000). These results can be explained with basis on the fact that the entropy change by swelling short polymer chains is larger than for long polymer chains [14]. This effect is alleviated in the MC as a larger curvature bending for the shorter polymer chains. 
The deswelling ramps showed that at $\sim 20 \% \mathrm{RH}$ the original position of PHEMA coated MCs was achieved. For RH values lower than $20 \%$ the MCs coated with PHEMA-20, PHEMA300 and PHEMA-1000 kept bending towards the coated side up to maximal values of $9 \pm 1 \mu \mathrm{m}$ at $\sim 2 \% \mathrm{RH}, 6.6 \pm 0.5 \mu \mathrm{m}$ at $\sim 2.5 \% \mathrm{RH}$ and $1.5 \pm 0.5 \mu \mathrm{m}$ at $\sim 3.5 \% \mathrm{RH}$, respectively. The tensile stress indicates that attractive forces dominate the intermolecular interaction on the MC surface. The polymer layer is expected to shrink at RH range of $2.5 \%$ to $3.5 \%$, while it adopts a more extended conformation at $0 \% \mathrm{RH}$. A similar behavior has been observed and theoretically modeled for self-assembled monolayers of ssDNA, a polymer with strong affinity for water molecules [6, 31, 32]. Rabin et al. [32] proposed a model to explain polyelectrolyte monolayers shrinking upon adsorbing water; they considered that at low concentrations of water vapor, the adsorption of water is accompanied by enhanced expulsion of vacancies, and thus, compression of the monolayer. Swelling and deswelling cycles were also performed for PS coated MCs as control experiments (Figure 3a). The deflections were negligible, indicating that the behavior observed for PHEMA-20, PHEMA-300 and PHEMA-1000 in Figure 3a arises due to the favorable interactions between $\mathrm{SiO}_{2} / \mathrm{PHEMA}, \mathrm{SiO}_{2} /$ water and PHEMA/water.

Figure $3 \mathrm{~b}$ shows the swelling and deswelling cycles determined for PHEMA-300, PS and PS/PHEMA-300 coated MCs. The deflections observed for PS/PHEMA-300 were much less pronounced than those observed for PHEMA-300. These results indicate the existence of a thick interfacial water layer between $\mathrm{SiO}_{2}$ (bare $\mathrm{MC}$ ) and PHEMA and a thin interfacial water layer between PS (deposited onto MC) and PHEMA that plays a substantial role on the signal transduction.

Figures $3 \mathrm{a}$ and $3 \mathrm{~b}$ show $\mathrm{MC}$ responses to humidity cycles of $15 \mathrm{~min}$. In order to evaluate the effect of period of time set for swelling and deswelling cycles on the MC deflections, the responses of PHEMA-300 coated MC were recorded for humidity cycles of 8 min, $15 \mathrm{~min}$ and $60 \mathrm{~min}$ (Figure 3c). The systems behaved similarly, indicating that the time needed to exchange the atmosphere in the environmental cell seems to be short in comparison to the periods of time between 8 and 60 min used for the humidity cycles.

Mechanical resonators are based on the change of the nanometer-scale vibration of a suspended MC when molecules adsorb on its surface. Mass changes can be determined accurately by following the $M C$ resonance frequency if (i) the elastic properties of the $M C$ remain unchanged during the molecules adsorption and (ii) damping effects are negligible [33]. The resonant frequency sensitively shifts in proportion to the mass of the molecules adsorbed on the MC. Moreover, the MC used in this study are homogeneously covered by the PS or PHEMA films (Figure 2) and considering that water absorption is also homogeneous in the 
entire film, the simple harmonic oscillator model can be applied to estimate the amount of water absorbed by the polymeric films when exposed to high humidity. Table 1 shows the difference in surface stress between the upper and lower sides of the MCs, $\Delta \sigma$, calculated by the modified Stoney's formula [29]. $\Delta \sigma$ is related to the microcantilever deflection, $\Delta z$, the length of the cantilever, L, Poisson's ratio, v, Young's modulus, E, and the thickness of the cantilever, h:

$$
\Delta z=\frac{3(1-v) L^{2}}{E h^{2}} \Delta a
$$

The resonance frequency was determined for each PHEMA coated MC at increasing RH ramp (see Supplementary Information Figure S2 for details). Table 1 shows the relative resonance frequency shift $(\Delta f)$, which is the difference between the $M C$ resonance frequency determined at $80 \% \mathrm{RH}$ and at $0 \% \mathrm{RH}$. The largest $\Delta \mathrm{f}$ value was observed for PHEMA-20 and the smallest one was for PHEMA-1000, corroborating the deflection behavior observed in Figure 3a. The negative $\Delta f$ values observed are related to mass increase $(\Delta \mathrm{m})$ due to swelling:

$$
\Delta f=\frac{1}{2 \pi} \sqrt{\frac{k}{\Delta m}}
$$

where $\mathrm{k}$ is the cantilever spring constant. The relative mass increases are presented in Table 1; the largest value was determined for PHEMA-20 coated MCs as $(5.1 \pm 0.8) \%$. The dependence of $\Delta f$ and relative mass increase values on PHEMA molecular weight is presented in Figures $4 \mathrm{a}$ and $4 b$, respectively. Although there are only three data points, $\Delta f$ and relative mass increase values suggest that PHEMA swelling tends to increase exponentially with the decrease of molecular weight. The $\Delta f$ and relative mass increase values determined for PHEMA-300 and PS/PHEMA-300 are comparable, although $\Delta \sigma$ values are quite different, indicating that the amount of water is approximately the same, but the water molecules at the interface $\mathrm{SiO}_{2}$ /PHEMA are arranged in a different way than at the interface PS/PHEMA. Moreover, the organization of water molecules in the system polymer/MC does not depend on the polymer layer thickness because PHEMA-300 and PHEMA-1000 have similar thicknesses, but presented quite different values of $\Delta \sigma, \Delta f$ and relative mass increase. PHEMA-300 was deposited onto PS coated MCs, but dewetting of PHEMA-20 films impeached their use in the deflection curves under humidity cycles (see Supplementary Information Figure S3).

Hysteresis observed for swelling and deswelling isotherms determined for PHEMA-20, PHEMA-300 and PHEMA-1000 occurs when the water content in deswelling at the same water activity is higher than in swelling, indicating water retention in the system polymer/MC [34]. At low $\mathrm{RH}$ the persistence length increases and then the entropy penalty is lower and thus the 
bending, at high RH chains explore a wider conformational space and thus the entropy penalty needs to be balanced by a larger bending.

The arrangement of interfacial water layer and polymer chain length seem to control the MCs deflections, since they result from differential surface stress generated between opposite sides of MCs. In the case of hydrophilic substrate, as $\mathrm{SiO}_{2}$, water interacts with both, substrate and PHEMA, by hydrogen bonding. The hydroxyl groups of PHEMA will tend to orient to the water wetted $\mathrm{SiO}_{2}$ surface, as schematically represented in Figure 5a. Such effect is favored for lower molecular weight PHEMA, which have smaller entropic constraints. On the other hand, hydrophobic substrates, as PS, are not wet by water and the PHEMA methyl groups are expected to be oriented to the substrate, driven by weak dispersion forces, as depicted in Figure $5 \mathrm{~b}$. Thus, the interfacial water layer between $\mathrm{SiO}_{2}$ (bare $\mathrm{MC}$ ) and PHEMA is more strongly bound than in the case of the interfacial water layer between PS (deposited onto $\mathrm{MC})$, resulting in different signal transduction.

Ellipsometry, an optical technique very sensitive to changes in thickness (d) and index of refraction ( $n$ ) of thin films, was used in order to obtain additional experimental evidences for the proposed model. For environmental conditions with $\mathrm{RH}<80 \%$, the changes in the ellipsometric angles ( $\Delta$ and $\psi$ ) measured for polymeric films were too small, even after $4 \mathrm{~h}$ in contact with humidity (Supplementary Information Figure S4). Ellipsometric measurements were successfully performed for PHEMA-300 at $80 \%$ RH as a function of time. Figures $6 a$ and $6 b$ show the linear dependence of mean $d$ and $n$ values determined for PHEMA-300 onto Siwafers as a function of square root of time. Such behavior is typical for Fickian diffusion mechanism, which describes the transport of molecules through matrix without any specific interactions between liquid molecule and polymer matrix. The fitting equations (4) and (5) allow estimating $d$ and $\mathrm{n}$ values for $15 \mathrm{~min}$ as being $358.5 \mathrm{~nm}$ and 1.4536, respectively. The increase in $d$ value corresponds to $1.3 \%$ of the initial value, which is due to the swelling. However, it is not possible to correlate directly with the amount of water in the PHEMA layer.

$$
\begin{aligned}
& d(n m)=353.7+1.2389 t^{0.5} \\
& n=1.4652-0.003 t^{0.5}
\end{aligned}
$$

The swelling degree can be estimated by the changes in the index of refraction of PHEMA layer:

$$
\begin{aligned}
& \mathrm{n}_{\mathrm{x}}=\mathrm{n}_{\text {PHEMA }} \varphi_{\text {PHEMA }}+\mathrm{n}_{\text {water }} \varphi_{\text {water }} \\
& \varphi_{\text {PHEMA }}+\varphi_{\text {water }}=1
\end{aligned}
$$


where $n_{x}$ is the index of refraction of a swollen PHEMA layer, $n_{\text {PHEMA }}$ and $n_{\text {water }}$ stand for the indices of refraction of PHEMA ( $n=1.465 \pm 0.005)$ determined just after spin-coating and of distilled water $(n=1.3334 \pm 0.0005)$ determined with an Abbé refractometer at $(24 \pm 1){ }^{\circ} \mathrm{C}$, respectively. $\varphi_{\text {PHEMA }}$ and $\varphi_{\text {water }}$ represent the volume fraction of PHEMA and water in the layer, respectively. Substituting $n_{x}$ by 1.4536 in equation (6) and combining with equation (7), the $\varphi_{\text {water }}$ value of $8.8 \%$ was calculated. It corresponds to approximately three fold the amount of water estimated by $\Delta f$ in Table 1 . On the other hand, exposing PHEMA-1000 during $4 \mathrm{~h}$ at $80 \% \mathrm{RH}$ led to changes in order of the accuracy of the ellipsometric measurements $\left(\Delta \pm 0.1^{\circ}\right.$ and $\psi \pm 0.05^{\circ}$ ), corroborating with the small deflections observed for MC coated with PHEMA1000. Ellipsometric measurements for PHEMA-20 were not successful because the films became inhomogeneous (Supplementary Information Figure S3) and the laser beam was scattered from the surface to all directions. The spin-coater environment was conditioned with dried $\mathrm{N}_{2}$, so that just after spin-coating the short PHEMA-20 chains, particularly those in the uppermost layer, have larger mobility to expose the hydrophobic methyl groups to the low humidity environment (Figure 7a) than the long PHEMA-300 or PHEMA-1000 chains. Upon contact with high $\mathrm{RH}$ in the ellipsometric cell, the hydroxyl groups tend to orientate to the humid air (Figure 7b), causing molecular segregation on the surface and creating the morphological inhomogeneity observed (Supplementary Information Figure S3).

It was not possible to monitor the thickness or the index of refraction evolution for any PHEMA films deposited onto PS coated Si wafers by ellipsometry. Either the PHEMA films dewetted or became too inhomogeneous (Supplementary Information Figure S3).

The limitations of ellipsometry in comparison to the detection of nanomechanical response are the (i) low optical contrast between $\mathrm{SiO}_{2}$ and $\mathrm{PHEMA}$, which restricted the experiments to substantial swelling common to high RH levels and (ii) films inhomogeneity. Thus, MC sensors are advantageous because physical changes in the polymeric films can be detected in a much shorter period of time and in a broad RH range, regardless the film morphology.

\section{Conclusions}

Microcantilevers sensors are excellent sensors of physical surface phenomena and have great potential as a sensitive tool for the rapid characterization of thin films of diverse materials. We have studied systematically the swelling and deswelling behavior of PHEMA films with three different average molecular masses deposited onto bare MCs or onto 
polystyrene (PS) coated MCs by monitoring the deflections resulting from differential surface stress under humidity cycles. We observe that PHEMA swelling tended to increase exponentially with the decrease of molecular weight, since short polymer chains have higher degree of freedom than long polymer chains. Ellipsometric measurements evidenced the swelling behavior of PHEMA-300 films under $80 \%$ RH as Fickian diffusion. However, due to the weak optical contrast or film homogeneity shorter or longer chains than PHEMA-300 could not be investigated by ellipsometry. Dewetting of PHEMA films deposited onto PS surfaces also impeached the ellipsometric studies. Therefore, in comparison to ellipsometry, monitoring swelling behavior of PHEMA by nanomechanical responses proved to be faster and less restricted.

\section{References}

1. A. Boisen, S. Dohn, S. S. Keller, S. Schmid and M. Tenje, Cantilever-like micromechanical sensors, Rep. Prog. Phys. 74 (2011) 036101 (30pp).

2. M. Dionisio, G. Oliviero, D. Menozzi, S. Federici, R. M. Yebeutchou, F. P. Schmidtchen, E. Dalcanale, P. Bergese, Nanomechanical Recognition of N-Methylammonium Salts, J. Am. Chem. Soc. 134 (2012) 2392-2398.

3. J. Tamayo, P. M. Kosaka, J. J. Ruz, A. San Paulo and M. Calleja, Biosensors based on nanomechanical systems. Chem. Soc. Rev. 42 (2013) 1287-1311.

4. M. Calleja, P. M. Kosaka, A. San Paulo and J. Tamayo, Challenges for nanomechanical sensors in biological detection, Nanoscale, 4 (2012) 4925-4938.

5. K. Gruber, T. Horlacher, R. Castelli, A. Mader, P. H. Seeberger and B. A. Hermann, Cantilever Array Sensors Detect Specific Carbohydrate-Protein Interactions with Picomolar Sensitivity, ACS Nano 5 (2011) 3670-3678.

6. J. Mertens, C. Rogero, M. Calleja, D. Ramos, J. A. Martin-Gago, C. Briones and J. Tamayo, Label-free detection of DNA hybridization based on hydration-induced tension in nucleic acid films, Nature Nanotechnol. 3 (2008) 301-307.

7. J. Fritz, M. K. Baller, H. P. Lang, H. Rothuizen, P. Vettiger, E. Meyer, H. J. Güntherodt, Ch. Gerber and J. K. Gimzewski, Translating biomolecular recognition into nanomechanics, Science 288 (2000) 316-318. 
8. R. McKendry, J. Zhang, Y. Arntz, T. Strunz, M. Hegner, H. P. Lang, M. K. Baller, U. Certa, E. Meyer, H. J. Guntherodt and Ch. Gerber, Multiple label-free biodetection and quantitative DNA-binding assay on a nanomechanical cantilever array, Proc. Natl. Acad. Sci USA 99(2002), 9783-9788

9. N. Backmann, C. Zahnd, F. Huber, A. Bietsch, A Pluckthun, H-P. Lang, H-J. Guntherodt, M. Hegner and Ch. Gerber, A label-free immunosensors array using single-chain antibody fragments, Proc. Natl. Acad. Sci. U. S. A., 102(2005), 14587-14592.

10. P. M. Kosaka, J. Tamayo, J. J. Ruíz, S. Puertas, E. Polo, V. Grazu, J. M. de la Fuente and M. Calleja, Tackling reproducibility in microcantilever biosensors: a statistical approach for sensitive and specific end-point detection of immunoreactions, Analyst 138 (2013) 863-872.

11. Y. Arnts, J. D. Seeling, H. P. Lang, J. Zhang, P. Hunziker, J. P. Ramseyer, E. Meyer, M. Hegner and Ch. Gerber, Label-free protein assay based on a nanomechanical cantilever array, Nanotechnology 14(2003) 86-90.

12. N. Jung, H. Seo, D. Lee, C. Y. Ryu and S. Jeon, Nanomechanical Thermal Analysis of the glass transition of polystyrene using silicon cantilevers, Macromolecules 41 (2008) 6873-6875.

13. O. Ahumada, M. M. Pérez-Madrigal, J. Ramirez, D. Curcó, C. Esteves, A. SalvadorMatar, G. Luongo, E. Armelin, J. Puiggalí, and C. Alemán, Sensitive thermal transitions of nanoscale polymer samples using the bimetallic effect: Application to ultra-thin polythiophene, Rev. Sci. Instr. 84 (2013) 053904.

14. G-G. Bumbu, M. Wolkenhauer, G. Kircher, J. S. Gutmann and Rüdiger Berger, Micromechanical Cantilever Technique: A Tool for Investigating the Swelling of Polymer Brushes, Langmuir 23 (2007) 2203-2207.

15. K. M. Holder, M. A. Priolo, K. E. Secrist, S. M. Greenlee, A. J. Nolte and J. C. Grunlan, Humidity-Responsive Gas Barrier of Hydrogen-Bonded Polymer-Clay Multilayer Thin Films. J. Phys. Chem. C 116 (2012) 19851-19856.

16. F. Doumenc, H. Bodiguel, and B. Guerrier, Physical aging of glassy PMMA/toluene films: Influence of drying/swelling history. Eur. Phys. J. E 27 (2008) 3-11. 
17. S-W. Lee and D. Lee, Integrated Study of Water Sorption/Desorption Behavior of Weak Polyelectrolyte Layer-by-Layer Films, Macromolecules 46 (2013) 2793-2799.

18. M. Ide, T. Mori, K. Ichikawa, H. Kitano, M. Tanaka, A. Mochizuki, H. Oshiyama and W. Mizuno, Structure of Water Sorbed into Poly(MEA-co-HEMA) films as Examined by ATR-IR Spectroscopy. Langmuir 19 (2003) 429-435.

19. S. Chatzandroulis, D. Goustouridis, I. Raptis, Polymeric film characterization for use in bimorph chemical sensors. Microelectronic Engineering 78-79 (2005) 118-124.

20. A. Opdahl, S. H. Kim, T. S. Koffas, C. Marmo, G. A. Somorjai. Surface mechanical properties of pHEMA contact lenses: Viscoelastic and adhesive property changes on exposure to controlled humidity. J. Biomed. Mater. Res. 67A (2003) 350-356.

21. T. S. Koffas, A. Opdahl, C. Marmo, G. A. Somorjai, Effect of Equilibrium Bulk Water Content on the Humidity-Dependent Surface Mechanical Properties of Hydrophilic Contact Lenses Studied by Atomic Force Microscopy. Langmuir 19 (2003) 3453-3460.

22. S. Igarashi, A. N. Itakura, M. Toda, M. Kitajima, L. Chu, A. N. Chifen, R. Förch, R. Berger, Swelling signals of polymer films measured by a combination of micromechanical cantilever sensor and surface plasmon resonance spectroscopy. Sensors and Actuators B 117 (2006) 43-49.

23. C. Liu, M. C. Lopes, S. A. Pihan, D. Fell, M. Sokuler, H.-J. Butt, G. K. Auernhammer, E. Bonaccurso, Water diffusion in polymer nano-films measured with microcantilevers, Sensors and Actuators B 160 (2011) 32-38.

24. N. F. Martinez, P. M. Kosaka, J. Tamayo, J. Ramírez, O. Ahumada, J. Mertens, T. D. Hien, C. V. Rijin and M. Calleja, High throughput optical readout of dense arrays of nanomechanical systems for sensing applications, Rev. Sci. Instr. 81 (2010) 125109.

25. R.M. Azzam, N.M. Bashara, Ellipsometry and Polarized Light, North Holland, Amsterdam, 1988.

26. Palik ED (1985) Handbook of optical constants of solids. Academic Press, London.

27. J. Fujimoto and D. F. S. Petri, Adsorption Behavior of Carboxymethylcellulose on Amino-Terminated Surfaces, Langmuir, 17, 56-60

28. D. Snow, B. L. Weeks, D. J. Kim, A. Loui, B. R. Hart, L. J. Hope-Weeks, Static deflection measurements of cantilever arrays reveal polymer film expansion and contraction. J. Coll. Int. Sci. 316 (2007) 687-693. 
29. C. Bradley, N. Jalili, S. K. Nett, L. Chu, R. Förch, J. S. Gutmann, R. Berger, Response characteristics of thermoresponsive polymers using nanomechanical cantilever sensors, Macromol. Chem. Phys. 210 (2009), 1339-1345.

30. T. Liu, S. Pihan, M. Roth, M. Retsch, U. Jonas, J. S. Gutmann, K. Koynov, H.-J. Butt and R. Berger, Frequency response of polmer films made from a precursor colloidal monolayer on a nanomechanical cantilever, Macromolecules 45 (2012) 862-871.

31. G.a Wu, H. Ji, K. Hansen, T. Thundat, R. Datar, R. Cote, M. F. Hagan, A. K. Chakraborty and A. Majumdar, Origin of nanomechanical cantilever motion generated from biomolecular interactions, Proc Natl Acad Sci U S A. 98 (2001) 1560-1564.

32. M. Wagaman, S. Medalion and Y. Rabin, Anomalous sweling of polymer monolayers by water vapour, Macromolecules 45 (2012) 9517-9521.

33. P. S. Waggoner and H. G. Craighead, Micro- and nanomechanical sensor for environmental, chemical and biological detection, Lab Chip, 2007, 7, 1238-1255.

34. Y. H. Roos, Water Activity - Principles and Measurements, in: Encyclopedia of Food Sciences and Nutrition, B. Cabellero, L. Trugo and P. Finglas (Eds.), Elsevier, London, 2003, pp. 6089-6094.

\section{Acknowledgments}

The authors acknowledge the financial support from the Brazilian Agency CNPq through the grants \#560565/2010-0 and \#209693/2012-6 and the financial support from the Spanish Science Ministry through projects \#PIB2010BZ-00510); and from European Research Council through Starting Grant NANOFORCELLS (ERC-StG-2011-278860). The authors acknowledge technical support from Salvador and O. Ahumada (MecWins) with the implementation of the automated RH control. 


\section{Figure Captions}

Scheme 1 - Main operation modes of nanomechanical sensors: (a) static mode, this mode can be designed to follow the mechanical response in real time and (b) dynamic mode.

Figure 1 - Bright field images of polymer films prepared by spin-coating onto microcantilevers (a) PS, (b) PHEMA-20, (c) PHEMA-300, (d) PS/PHEMA-300 and (e) PHEMA-1000.

Figure 2 - Scheme of the experimental setup used for the experiments. The MC chip is mounted in a 2D voice-coil actuator for the displacement of the chip in the $X$ and $Y$ axe direction. The out-of-plane displacement of the mechanical system is along the $Z$ axis. The incident laser beam is oriented orthogonally to the reflected laser beam and with one axis in the $\mathrm{X}-\mathrm{Z}$ plane. The system is also an environmental chamber and automated $\mathrm{RH}$ and temperature control.

Figure 3 - Microcantilever response to humidity cycle of 15 min: (a) PS, PHEMA-20, PHEMA200, PHEMA-1000 and (b) PHEMA-300, PS/PHEMA-300, PS. (c) PHEMA-300 coated MC response to humidity cycles of $8 \mathrm{~min}, 15 \mathrm{~min}$ and $60 \mathrm{~min}$. PS covered MCs were used as reference sample due to its hydrophobicity.

Figure 4 - Dependence of (a) microcantilever frequency shift ( $\Delta f$ ) and (b) relative mass increase values on PHEMA molecular weight due to an increase on the relative humidity (R.H.). The MC resonance frequency was determined at $80 \%$ R.H. and at $0 \%$ R.H.

Figure 5 - Scheme of the arrangement of interfacial water layer and polymer chain length at high and low R.H. when (a) PHEMA thin film was deposited onto a hydrophilic substrate $\left(\mathrm{SiO}_{2}\right)$ and (b) PHEMA thin film was deposited onto a hydrophobic substrate (PS). The gray rectangle represents the silicon microcantilever, the blue cycles are the water molecules, the green lines are represents the polymer PHEMA film and the orange line is the PS.

Figure 6 - Dependence of mean (a) thickness, d, and (b) index of refraction, $n$, as a function of square root of time determined for PHEMA-300 at $80 \%$ R.H. and $(24 \pm 1)^{\circ} \mathrm{C}$.

Figure 7-Schematic representation of orientation of methyl and hydroxyl groups of PHEMA at (a) low R.H. and (b) high R.H. 
Table 1 - Difference in surface stress between the upper and lower sides of the MCs calculated using eq. (1) for maximal $\Delta \mathrm{z}, \Delta \sigma_{\max }$; the relative frequency shift $(\Delta f)$ which is the difference between the resonance frequency determined at $80 \% \mathrm{RH}$ and at $0 \%$ $\mathrm{RH}$; relative mass increase determined with $\Delta f$ and eq. (2).

\begin{tabular}{|l|c|c|c|c|}
\hline \multicolumn{1}{|c|}{ Coating } & $\Delta \boldsymbol{\sigma}_{\max }(\mathbf{N} / \mathbf{m})^{*}$ & $\boldsymbol{\Delta f}(\mathbf{k H z})$ & $\begin{array}{c}\text { Relative mass } \\
\text { increase (\%) }\end{array}$ & $\begin{array}{c}\text { Thickness } \\
(\mathbf{n m})\end{array}$ \\
\hline PHEMA-20 & $10 \pm 1$ & $-(0.20 \pm 0.03)$ & $5.1 \pm 0.8$ & $106 \pm 4$ \\
\hline PHEMA-300 & $7 \pm 1$ & $-(0.13 \pm 0.02)$ & $3.2 \pm 0.4$ & $220 \pm 14$ \\
\hline $\begin{array}{l}\text { PS/PHEMA- } \\
\text { 300 }\end{array}$ & $0.9 \pm 0.3$ & $-(0.12 \pm 0.04)$ & $2.9 \pm 0.6$ & $207 \pm 10$ \\
\hline PHEMA-1000 & $1.4 \pm 0.3$ & $-(0.06 \pm 0.01)$ & $1.5 \pm 0.4$ & $233 \pm 17$ \\
\hline PS & $0.004 \pm 0.003$ & $-(0.003 \pm 0.002)$ & $0.09 \pm 0.06$ & $223 \pm 15$ \\
\hline
\end{tabular}

*calculated for the maximal $\Delta \mathrm{z}, \mathrm{E}=160 \mathrm{GPa}, v=0.22, \mathrm{~L}=400 \mu \mathrm{m}$ and $\mathrm{h}=1.0 \mu \mathrm{m}$.

Temperature was kept constant at $(25 \pm 1){ }^{\circ} \mathrm{C}$. 
Ms. Mercedes del Rey - Degree in electronics engineering. She is working on the characterization of polymer films under humidity and temperature cycles by monitoring the deflections of microcantilevers resulting from differential surface stress. She is also interested in the development of optomechanical systems. She is a member of the Bionanomechanics Lab in the Microelectronics Institute of Madrid since 2010.

Mr. Rubens Araujo Silva - Ph.D. Student at the University of Sao Paulo since 2010. His Ph.D. thesis focuses on interfacial phenomena in thin layers of macromolecules. He has co-authored four publications.

Dr. David Meneses Rodríguez - He received his BSc degree from the Universidad Autónoma de San Luis Potosí (Mexico) in 2001, in 2003 his MSc from CINVESTAV (Querétaro, México) and he obtained his PhD degree in Applied Sciences in the option of Nanosciences and Nanotechnology, from IPICYT ( San Luis Potosí, México) in 2009. Since September 2009 he has joined to the IMM as a postdoctoral researcher, where he has integrated in the Magnetoplasmonic group. His research interests focus on engineering novel nanostructures with magneto-plasmonic properties. He is an expert in the fabrication of nanostructures using colloidal lithography.

Prof. Dr. Denise Freitas Siqueira Petri - Full professor in Physical Chemistry at the University of Sao Paulo since 2014. Research in her group arises from her interest in synthetic and natural macromolecules at interfaces. The main focus is the fundamental understanding on the interaction between proteins, enzymes and polymeric thin films. The experimental tools are ellipsometry, atomic force microscopy, contact angle measurements and non-linear spectroscopic methods. She has published more than 110 papers, 4 book chapters and filled 4 patents.

Dr. Montserrat Calleja - Senior Researcher at the Microelectronics Institute of Madrid-CSIC (Spain), where she has been head of Dept. of Devices, Sensors and Biosensors from 2008-2012. Since 2004 she has focused her research in nanomechanical sensing, developing new instrumentation and technologies for the application of nanomechanics to Biology. She has co-authored more than 60 publications, leaded more than 10 research projects and filled 8 patents. In 2008 she launched the technological company Mecwins S.L. that pioneers the commercialization of nanomechanical sensors for clinical diagnosis. Her present research interests are in the study of the mechanical properties of living cells. In this field she has received an European Research Council Starting Grant in 2011. She was awarded the Miguel Catalan research prize in 2012.

Dr. Javier Tamayo - PhD in Physics by the University Complutense of Madrid in 1998. Postdoctoral Marie Curie fellow at the University of Bristol (UK), developing SPM techniques in Biology. Since 2001 he has been a Research Staff member at the IMM/CNM/CSIC and he is leading the Bionanomechanics Group, devoted to the application of micro- nanomechanical structures for biological interfacing. He has 6 licensed patents and has published 77 articles in indexed journals, which have received more than 3600 citations. His h-index is 30 . He has leaded more than ten national and international projects along the last five years. Javier Tamayo is scientific promoter and founder of the spin-off company Mecwins S.L., fully devoted to the development of nanomechanical sensors for biomedicine.

Dr. Priscilla Monteiro Kosaka - PhD in Physical Chemistry by the University of Sao Paulo (Brazil) in 2008. Her Ph.D. thesis was focused on the thermodynamic stability of thin polymer films. The main goal during her Ph.D. was the preparation of cellulose derivatives thin films for biomedical applications. Since 2008 she is a member of the Bionanomechanics Lab in the Microelectronics Institute of Madrid. She believes that chemistry at nanoscale can lead to better understanding of biological processes and her principal interests are biosensing and biotechnology. She is responsible for the activities related to surface functionalization in the actual group and works on the optimization of immobilization strategies on 
microcantilevers for biosensing. She has co-authored more than 25 publications, 2 book chapters and filled 3 patents. 
Table 1 - Difference in surface stress between the upper and lower sides of the MCs calculated using eq. (1) for maximal $\Delta z, \Delta \sigma_{\max }$ the relative frequency shift $(\Delta f)$ which is the difference between the resonance frequency determined at $80 \% \mathrm{RH}$ and at $0 \% \mathrm{RH}$; relative mass increase determined with $\Delta f$ and eq. (2).

\begin{tabular}{|c|c|c|c|c|}
\hline Coating & $\Delta \sigma_{\max }(\mathrm{N} / \mathrm{m})^{*}$ & $\Delta f(\mathrm{kHz})$ & $\begin{array}{l}\text { Relative mass } \\
\text { increase (\%) }\end{array}$ & Thickness (nm) \\
\hline PHEMA-20 & $10 \pm 1$ & $-(0.20 \pm 0.03)$ & $5.1 \pm 0.8$ & $106 \pm 4$ \\
\hline PHEMA-300 & $7 \pm 1$ & $-(0.13 \pm 0.02)$ & $3.2 \pm 0.4$ & $220 \pm 14$ \\
\hline PS/PHEMA-300 & $0.9 \pm 0.3$ & $-(0.12 \pm 0.04)$ & $2.9 \pm 0.6$ & $207 \pm 10$ \\
\hline PHEMA-1000 & $1.4 \pm 0.3$ & $-(0.06 \pm 0.01)$ & $1.5 \pm 0.4$ & $233 \pm 17$ \\
\hline PS & $0.004 \pm 0.003$ & $-(0.003 \pm 0.002)$ & $0.09 \pm 0.06$ & $223 \pm 15$ \\
\hline
\end{tabular}

*calculated for the maximal $\Delta \mathrm{z}, \mathrm{E}=160 \mathrm{GPa}, \mathrm{v}=0.22, \mathrm{~L}=400 \mu \mathrm{m}$ and $\mathrm{h}=1.0 \mu \mathrm{m}$. Temperature was kept constant at $(25 \pm 1)^{\circ} \mathrm{C}$. 


\section{Scheme 1}

(a) Static mode

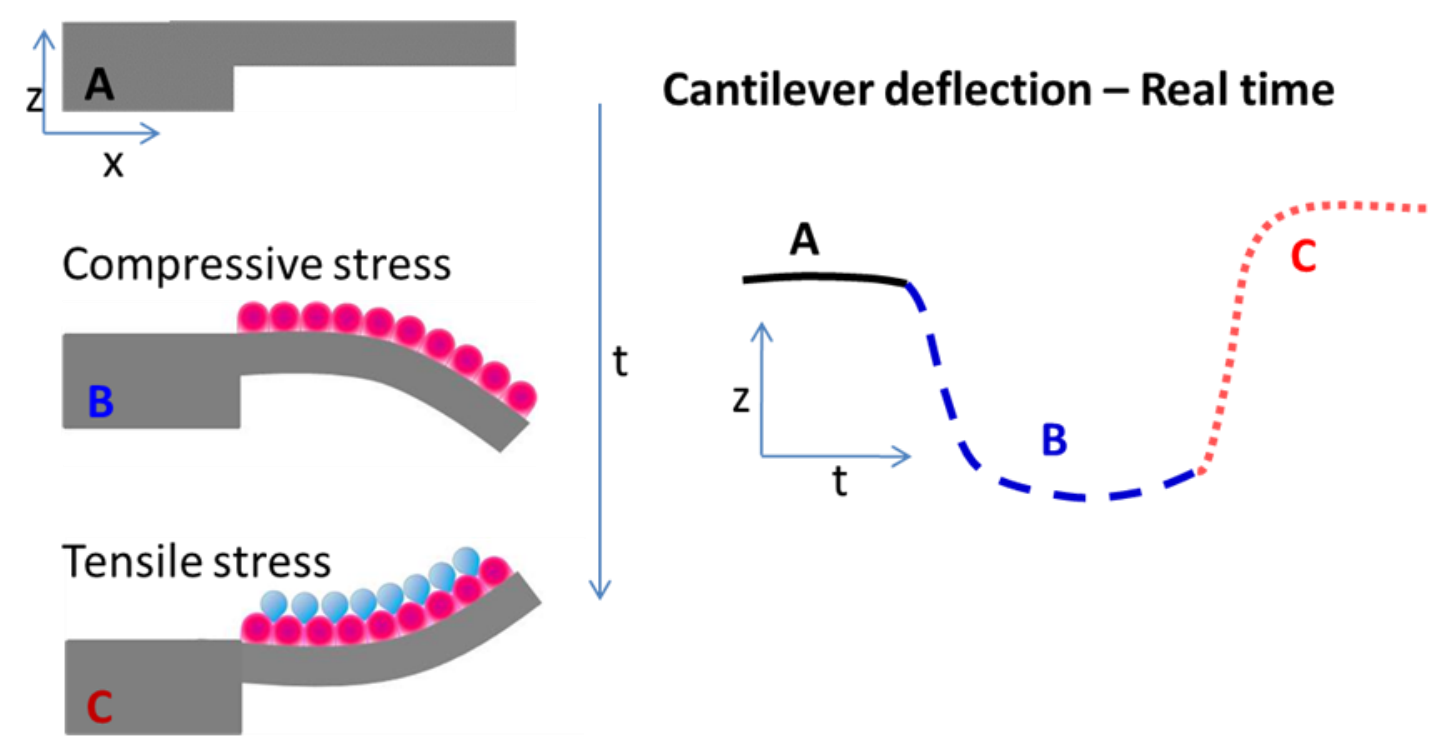

(b) Dynamic mode Added mass
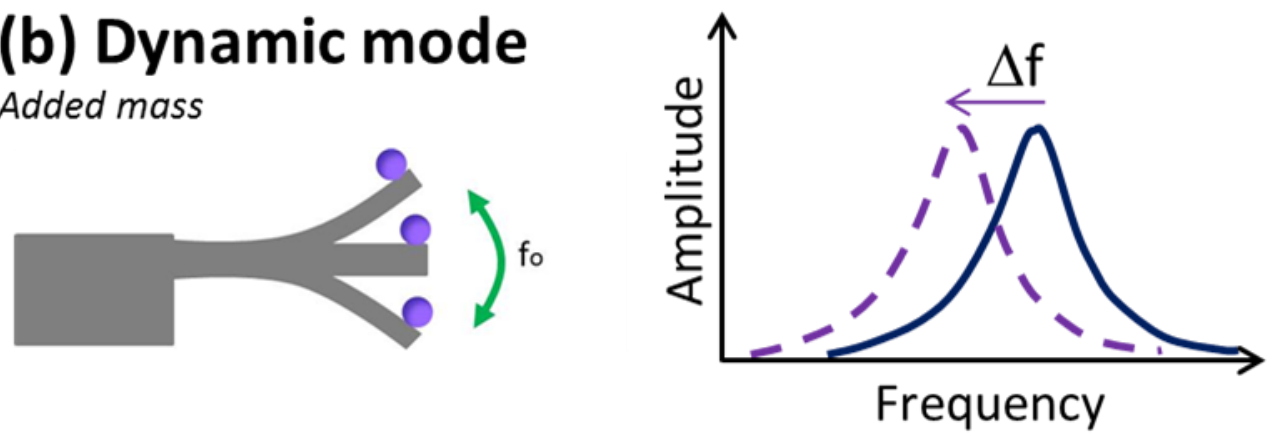
Figure 1

(a)

(b)

(c)

(d)

(e)
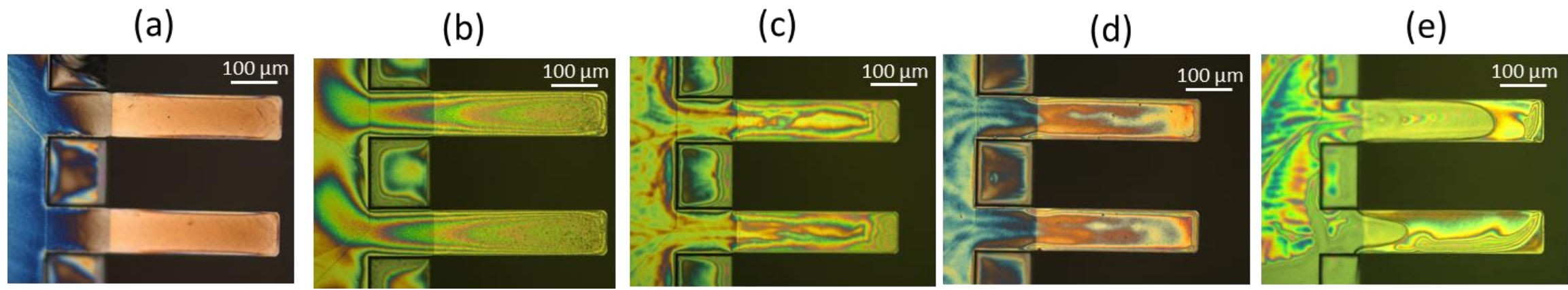


\section{Figure 2}

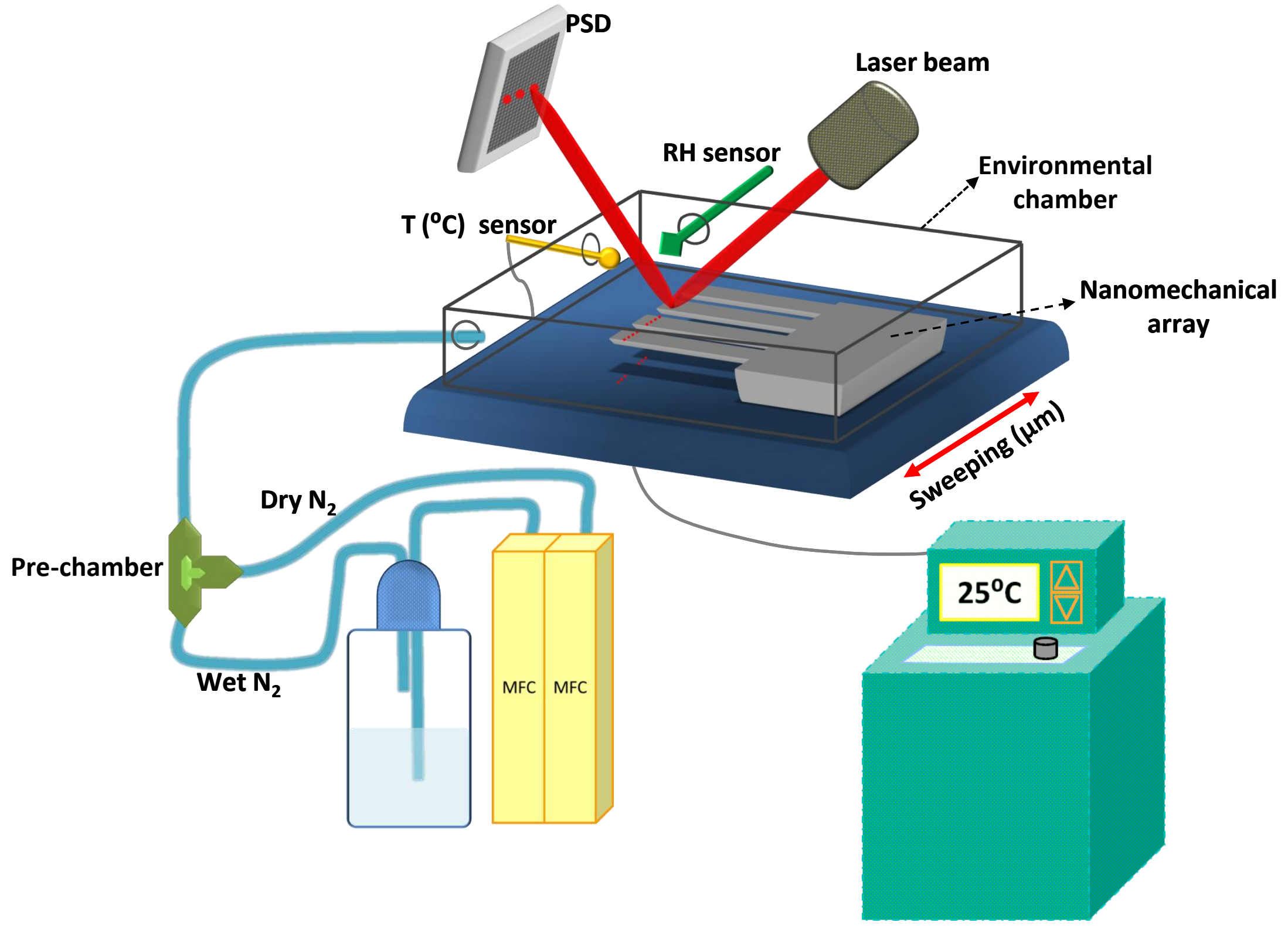




\section{Figure 3}

(a)

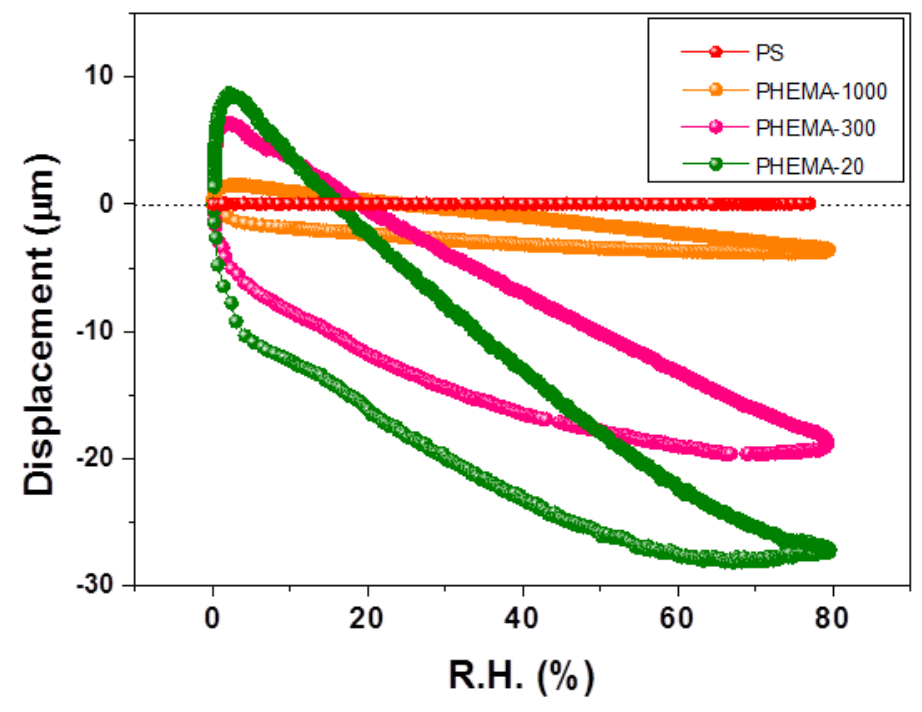

(b)

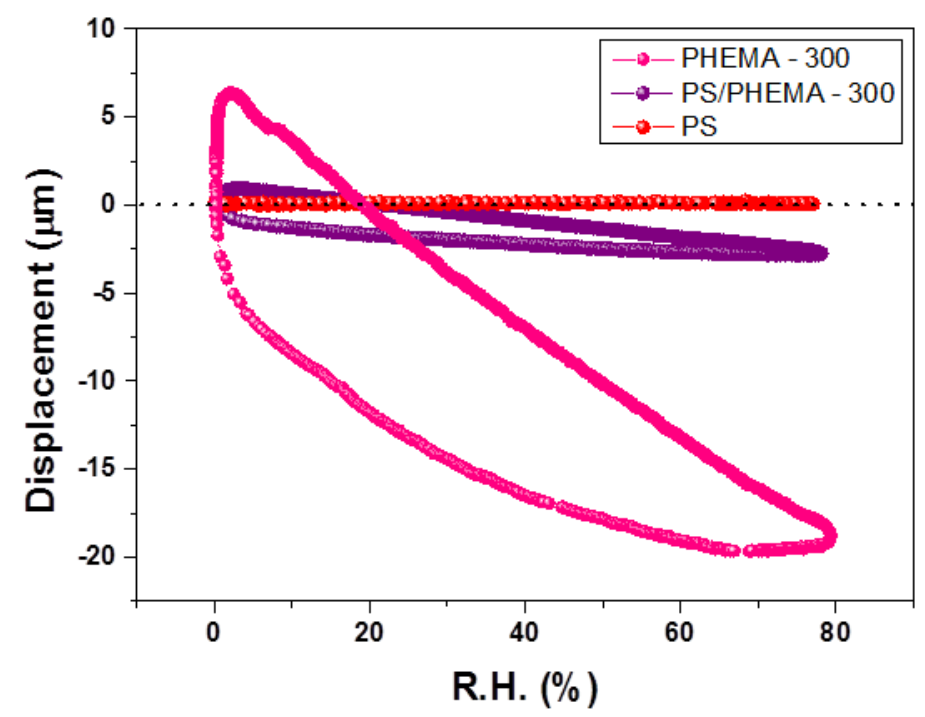

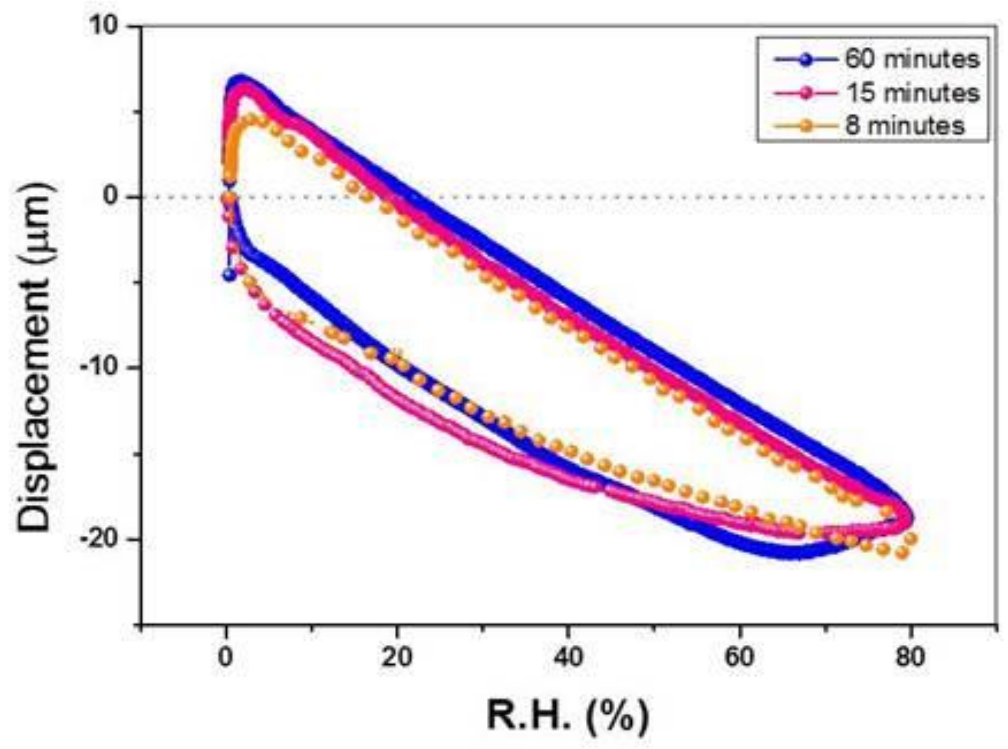

(c) 


\section{Figure 4}

(a)

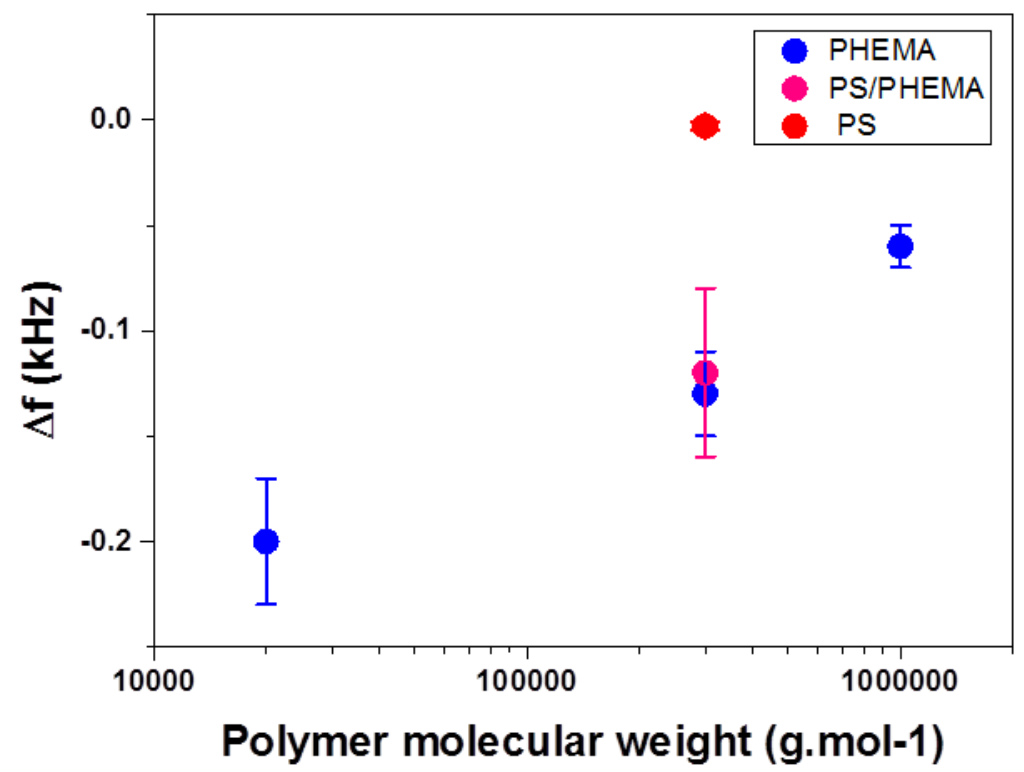

(b)

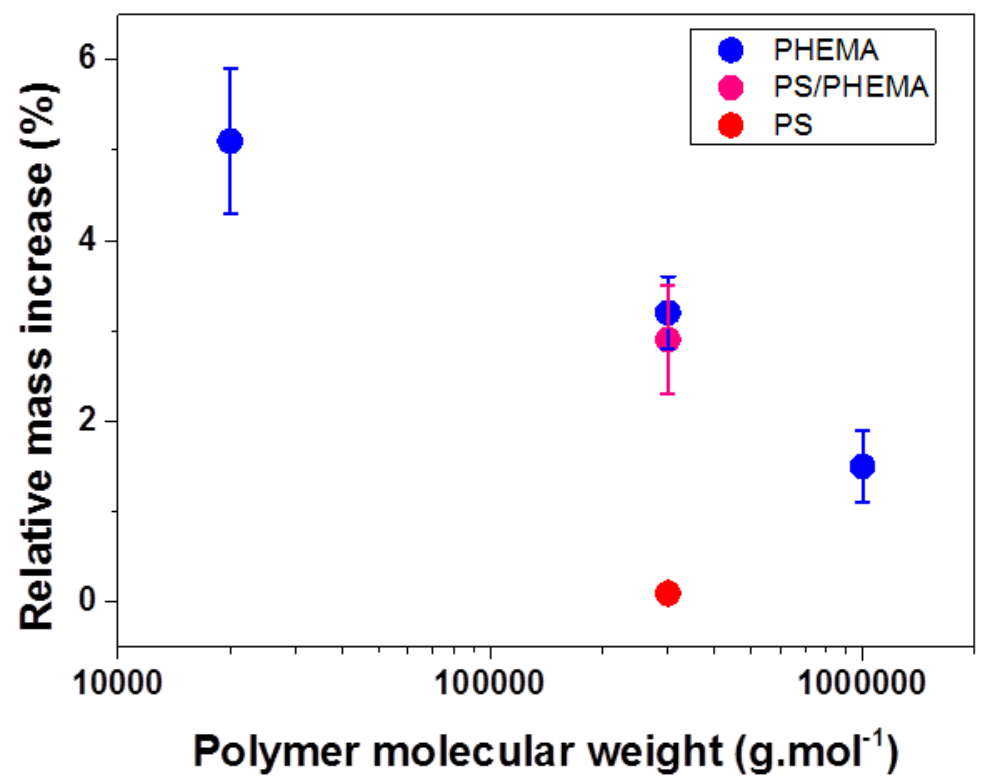


Figure 5a

Low R.H.

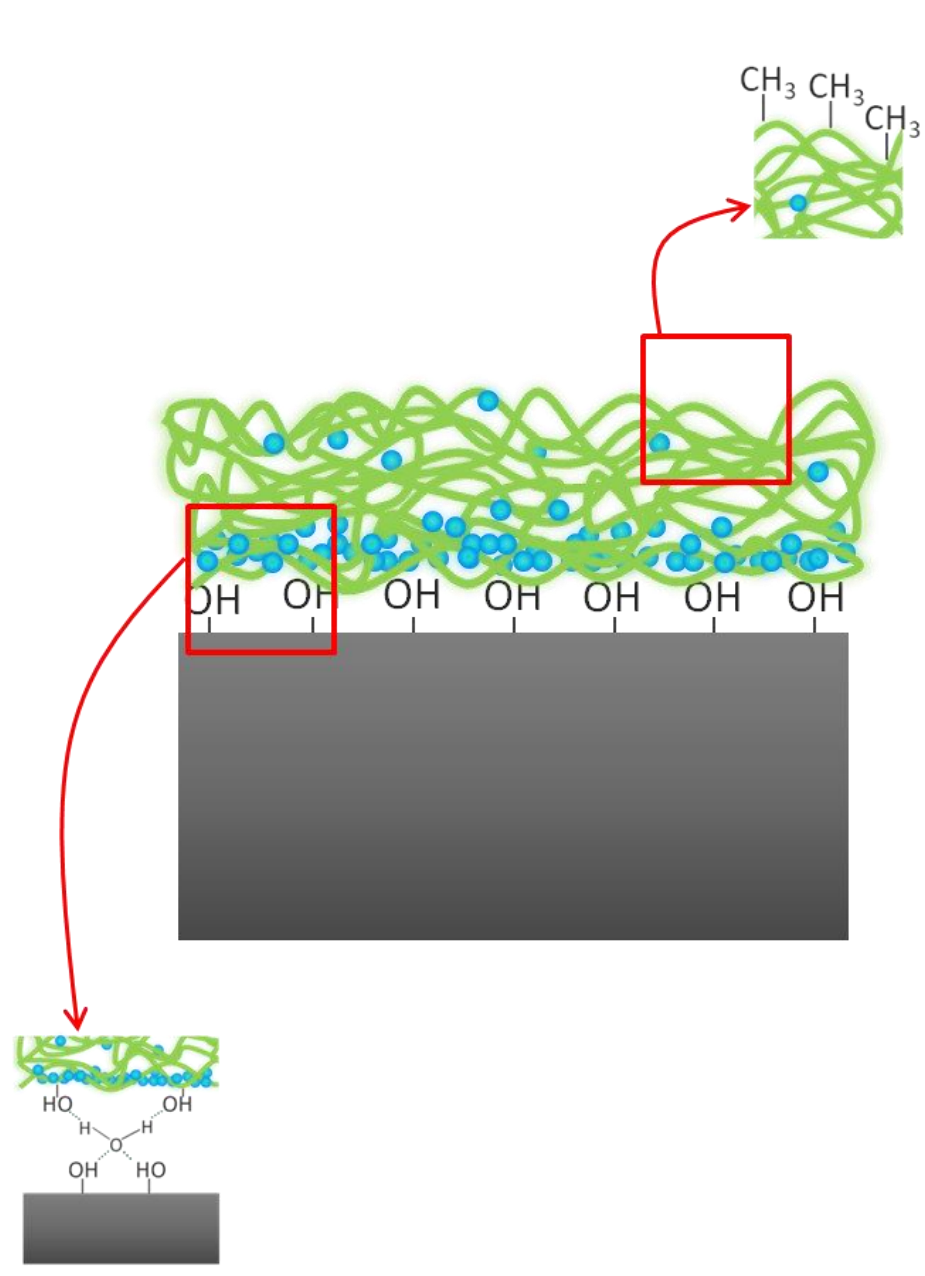

High R.H.

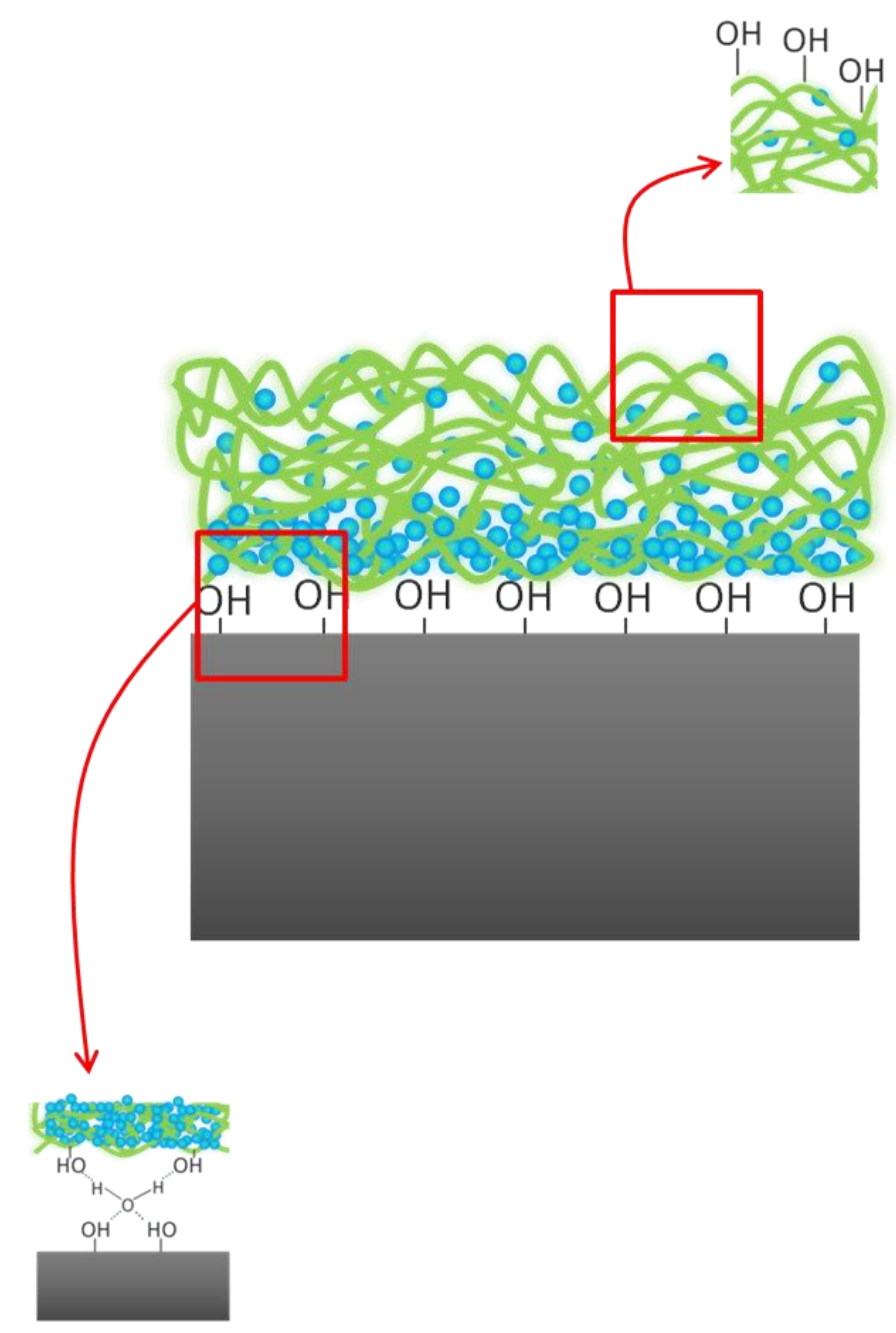




\section{Figure 5b}

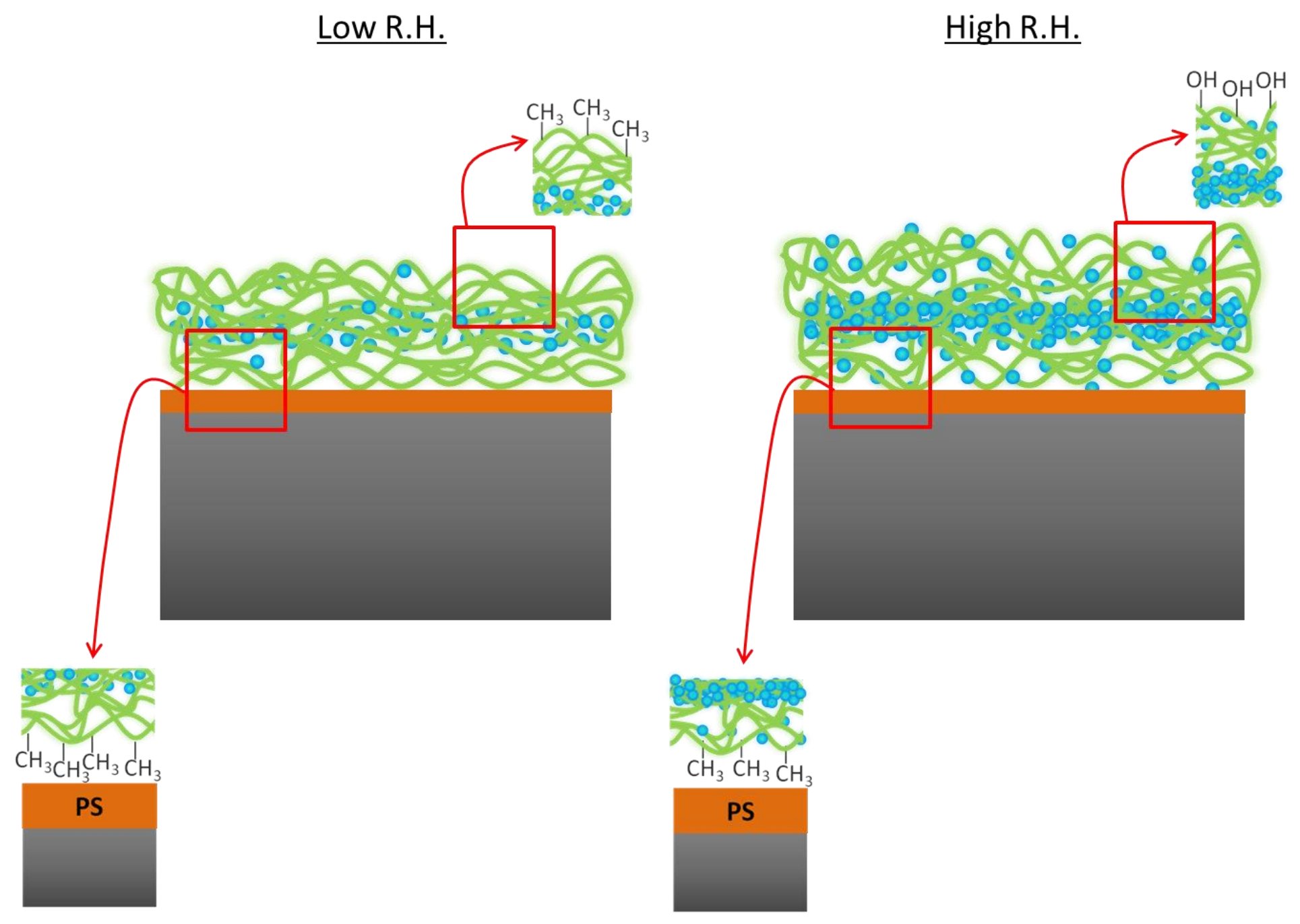


Figure 6a

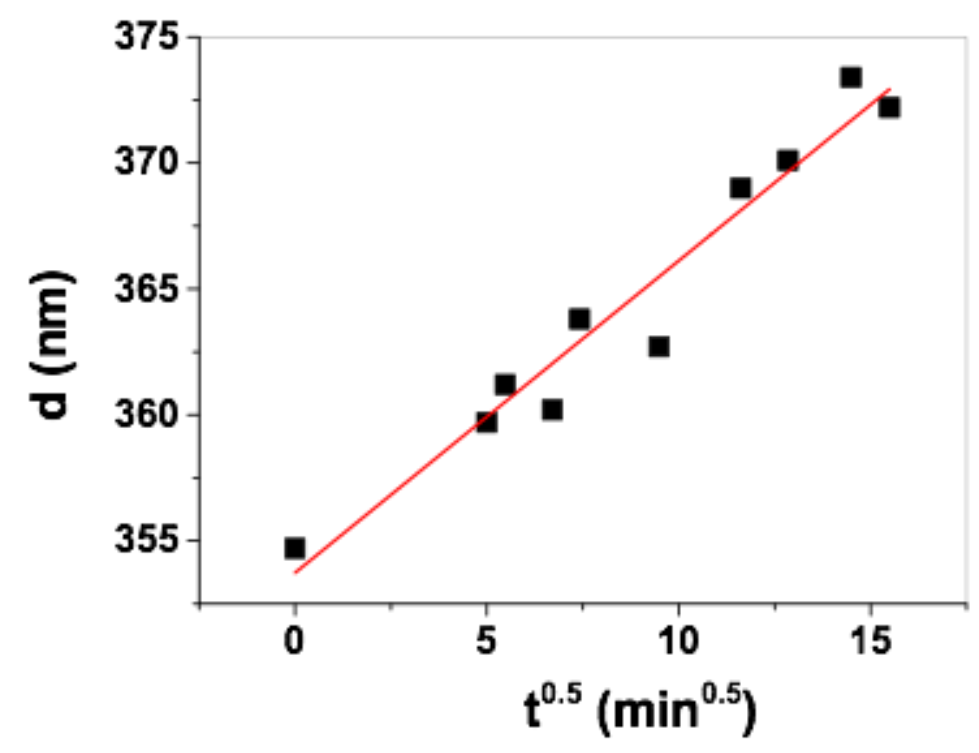

Figure 6b

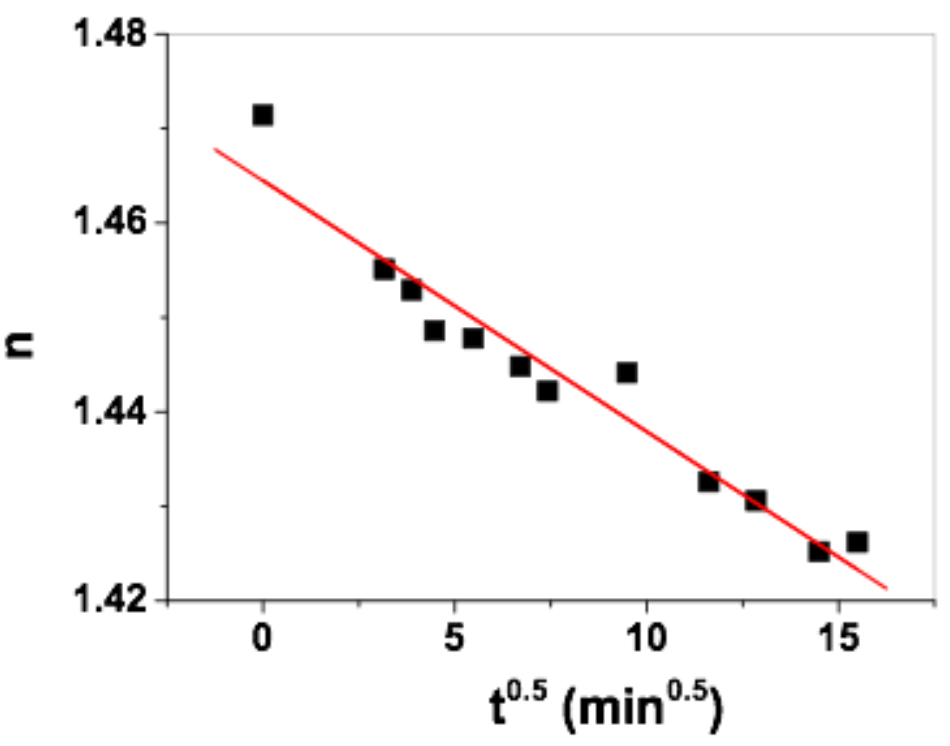


Figure 7

(a)

\section{Low RH}

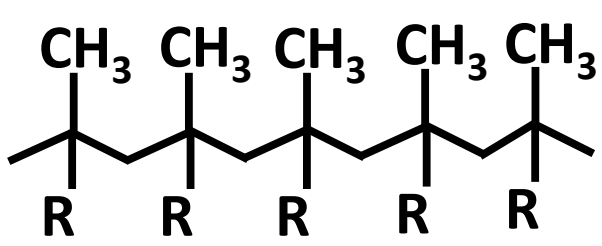

$\mathrm{R}=0 \nabla_{\mathrm{C}}-0 \sim_{\mathrm{OH}}$ (b)

High RH

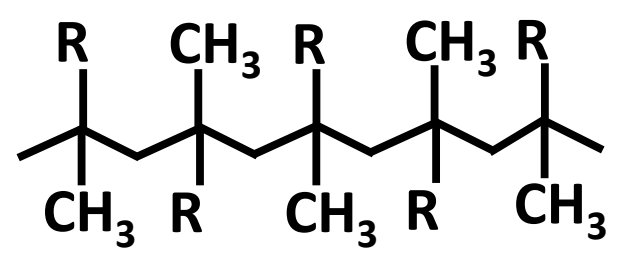

